\title{
ON THERMODYNAMICS OF RATIONAL MAPS ON THE RIEMANN SPHERE
}

\author{
TIEN-CUONG DINH, VIÊT-ANH NGUYÊN, AND NESSIM SIBONY
}

\begin{abstract}
We show that there is a spectral gap for the transfer operator associated to a rational map $f$ on the Riemann sphere. Using this and the method of pertubed operators we establish the (Local) Central Limit Theorem for the measure of maximal entropy of $f$, with an estimate on the speed of convergence.
\end{abstract}

\section{INTRODUCTION}

Let $f: \mathbb{P}^{1} \rightarrow \mathbb{P}^{1}$ be a rational map of degree $d \geq 2$. The Riemann sphere $\mathbb{P}^{1}$ is endowed with the Fubini-Study form $\omega$, normalized by $\int_{\mathbb{P 1}} \omega=1$. It is wellknown that the sequence $d^{-n} f^{n *}(\omega)$ converges weakly to a probability measure $\mu$ which is totally invariant: $d^{-1} f^{*}(\mu)=f_{*} \mu=\mu$, mixing, and which maximizes the entropy. This is the equilibrium measure of $f$. See Lyubich [19] or [24, 5, 6] for the multidimensional analogue.

The classical Birkhoff ergodic theorem implies that if $\phi$ is a $\mu$-integrable function then the Birkhoff sum $\frac{1}{n} \sum_{i=0}^{n-1} \phi \circ f^{i}$ converges $\mu$-almost everywhere ( $\mu$-a.e. for short) to $\mu(\phi)$. Assume that $\mu(\phi)=0$. Then, the Birkhoff sum converges $\mu$-a.e to 0 .

Recall that $\phi$ is a coboundary if there exists a function $\psi \in L^{2}(\mu)$ such that $\phi=\psi \circ f-\psi$. One easily checks that for such a function $\frac{1}{\sqrt{n}} \sum_{i=0}^{n-1} \phi \circ f^{i}$ converges to $0 \mu$-almost surely. We say that a real-valued function $\phi \in L^{1}(\mu)$ satisfies the Central Limit Theorem (CLT for short) if $\frac{1}{\sqrt{n}} \sum_{i=0}^{n-1} \phi \circ f^{i}$ converges in law to a Gaussian random variable of zero mean and variance $\sigma>0$. That is, for every interval $I \subset \mathbb{R}$,

$$
\lim _{n \rightarrow \infty} \mu\left\{\frac{1}{\sqrt{n}} \sum_{i=0}^{n-1} \phi \circ f^{i} \in I\right\}=\frac{1}{\sqrt{2 \pi} \sigma} \int_{I} e^{-\frac{t^{2}}{2 \sigma^{2}}} \mathrm{~d} t .
$$

Such a function is not a coboundary.

Let $\Lambda$ denote the adjoint of $f^{*}$ acting on $L^{2}(\mu)$. Sometimes, $\Lambda$ is called the Perron-Frobenius operator. We have

$$
\Lambda \phi(z)=\frac{1}{d}\left(f_{*} \phi\right)(z)=\frac{1}{d} \sum_{f(w)=z} \phi(w), \quad z \in \mathbb{P}^{1} .
$$

Date: February 20, 2006.

2000 Mathematics Subject Classification. Primary 37A25, 37F, Secondary 32U40, 32H50.

Key words and phrases. (Local) Central Limit Theorem, spectral gap. 
Consider a real-valued function $\phi \in L^{\infty}(\mu)$ such that $\mu(\phi)=0$, which is not a coboundary. A classical theorem of Gordin-Liverani [13, 18, implies that if

$$
\sum_{n \geq 0}\left\|\Lambda^{n} \phi\right\|_{L^{1}(\mu)}<\infty
$$

then $\phi$ satisfies the CLT with

$$
\sigma^{2}:=-\int_{\mathbb{P}^{1}} \phi^{2} \mathrm{~d} \mu+2 \sum_{n=0}^{\infty} \int_{\mathbb{P}^{1}} \phi\left(\phi \circ f^{n}\right) \mathrm{d} \mu=\lim _{n \rightarrow \infty} \int_{\mathbb{P}^{1}}\left(\frac{1}{\sqrt{n}} \sum_{k=0}^{n-1} \phi \circ f^{k}\right)^{2} \mathrm{~d} \mu .
$$

When $\phi$ is smooth, condition (1.2) is a direct consequence of the exponential decay of correlations proved by Fornæss and the third named author in [12. The case of Hölder continuous observables follows by interpolation between Banach spaces [8, 6], see also [5, 7] for the case of meromorphic maps in higher dimension. Note that with a completely different method (analysis of inverse branches) Haydn [14] has proved the exponential decay of correlations, hence the CLT, for Gibbs measures. A weaker result was previously proved by Denker, Przytycki and Urbański [4.

In this work, we study the speed of convergence in the CLT and prove the Local CLT. The approach is spectral theory for pertubation of the Perron-Frobenius operator $\Lambda$ in appropriate Sobolev spaces. We follow the Nagaev method [20, which was used by Rousseau-Egèle [23] and Broise [3. The main point here is to prove some necessary estimates in order to apply the Nagaev method. It turns out that the analogous estimates in the higher dimension case are more difficult. We also use a version of the classical result of Ionescu Tulcea and Marinescu.

We close this introduction with a brief outline of the paper to follow. In Section 2 we collect some background on Sobolev spaces. In Section 3 we develop the method of spectral theory for pertubation of Perron-Frobenius operator. This will enable us to prove the CLT (Theorem A) and obtain an estimate on the speed of convergence (Theorem B). Section 4 develops auxiliary tools used in Section 5 to prove the Local CLT (Theorem C).

Notation. For a normed-space $E$, denote by $\|\cdot\|_{E}$ its norm. We write $L^{p}$ instead of $L^{p}\left(\mathbb{P}^{1}\right)$, where the $L^{p}$-norm of a form is the sum of its coefficients for a fixed atlas of $\mathbb{P}^{1}$. If $\alpha_{10}, \alpha_{01}$ are forms of bidegrees $(1,0)$ and $(0,1)$ respectively, and $\alpha=\alpha_{10}+\alpha_{01}$, we use the equivalent $L^{2}$-norm $\|\alpha\|_{L^{2}}=\int_{\mathbb{P}^{1}}\left(i \alpha_{10} \wedge \bar{\alpha}_{10}+i \bar{\alpha}_{01} \wedge \alpha_{01}\right)$. If $T: E \rightarrow E$ is a linear operator, we denote by $\rho(T)$ its spectral radius. We also denote by $\Lambda$ the operator $\frac{1}{d} f_{*}$ even when it acts on forms. For a Radon measure $\nu,\|\nu\|$ is its total variation. The constants $C, C^{\prime}, C^{\prime \prime}$ and $C_{\epsilon}$ are not necessarily the same at each occurence; they depend possibly on some parameters.

\section{Sobolev SPACES}

The Sobolev space $W^{1, p}, p \geq 1$, is the space of complex-valued functions $\phi$ in $L^{p}$ such that $\mathrm{d} \phi$, which is defined in the sense of currents, has $L^{p}$ coefficients, see [1, 15]. It is endowed with the canonical norm $\|\phi\|_{W^{1, p}}=\|\phi\|_{L^{p}}+\|\mathrm{d} \phi\|_{L^{p}}$. Lipschitz functions belong to $W^{1, p}$. Let $\mathcal{L}$ denote the Lebesgue measure of mass 1 on $\mathbb{P}^{1}$ associated to the Fubini-Study form $\omega$. Define for each $L^{1}$ function its mean value 
$m(\phi):=\int_{\mathbb{P} 1} \phi \mathrm{d} \mathcal{L}$. We have the following Poincaré-Sobolev inequality: for every real number $p \geq 1$, there exists a constant $C=C(p)$ such that

$$
\left(\int_{\mathbb{P}^{1}}|\phi-m(\phi)|^{p} \mathrm{~d} \mathcal{L}\right)^{\frac{1}{p}} \leq C\|\mathrm{~d} \phi\|_{L^{2}}, \quad \phi \in W^{1,2} .
$$

In particular,

$$
\|\phi\|_{L^{p}} \leq|m(\phi)|+C\|\mathrm{~d} \phi\|_{L^{2}} \leq\|\phi\|_{L^{1}}+C\|\mathrm{~d} \phi\|_{L^{2}} \leq C^{\prime}\|\phi\|_{W^{1,2}} .
$$

Hence, $W^{1,2} \subset L^{p}$. Moreover, by Sobolev embedding theorem, the latter inclusion is compact. For every $\epsilon>0$, there is a constant $C=C(\epsilon)$ such that

$$
\|\phi\|_{L^{\infty}} \leq C\|\phi\|_{W^{1,2+\epsilon}}, \quad \phi \in W^{1,2+\epsilon},
$$

and

$$
\|\phi \psi\|_{W^{1,2+\epsilon}} \leq C\|\phi\|_{W^{1,2+\epsilon}}\|\psi\|_{W^{1,2+\epsilon}}, \quad \phi, \psi \in W^{1,2+\epsilon},
$$

(see, for example, Theorem 4.39 in [1]).

We recall from Lemmas 5.3 and 5.4 in [7] that

$$
|\mu(\phi)| \leq C\|\phi\|_{W^{1,2}}
$$

and

$$
\left\|\Lambda^{n} \phi-\mu(\phi)\right\|_{W^{1,2}} \leq \frac{C}{d^{n / 2}}\|\phi\|_{W^{1,2}}
$$

for every continuous $\phi \in W^{1,2}$ and $n \geq 0$. Since smooth functions are dense in $W^{1,2}$ we can extend $\mu$ to a continuous linear form of $W^{1,2}$ in a canonical way. Therefore, (2.5) -(2.6) are still valid for any $\phi \in W^{1,2}$. Moreover, $\mu$ is strongly continuous (WPC in the sense of [7]), i.e. if $\phi_{n} \rightarrow \phi$ weakly and $\left\|\phi_{n}\right\|_{W^{1,2}}$ is bounded then $\mu\left(\phi_{n}\right) \rightarrow \mu(\phi)$. It follows that one can associate to each function in $W^{1,2}$ a function in $L^{1}(\mu)$ in a canonical way.

Proposition 2.1. The following norm

$$
\|\phi\|:=|\mu(\phi)|+\|\mathrm{d} \phi\|_{L^{2}}
$$

is equivalent to the standard norm of $W^{1,2}$.

Proof. Inequality (2.5) implies that $\|\phi\| \leq C\|\phi\|_{W^{1,2}}$. From (2.2), we only need to check that there is a constant $C$ such that

$$
|m(\phi)| \leq|\mu(\phi)|+C\|\mathrm{~d} \phi\|_{L^{2}}, \quad \phi \in W^{1,2} .
$$

To prove this, observe that $m(\phi-m(\phi))=0$. Therefore, by (2.5),

$$
\left|\int_{\mathbb{P}^{1}}(\phi-m(\phi)) \mathrm{d} \mu\right| \leq C\|\mathrm{~d} \phi\|_{L^{2}}
$$

which implies (2.7). 


\section{Central Limit Theorem and speed of Convergence}

For every $\theta \in \mathbb{C}$ and every real-valued function $h$, consider the pertubation of the Perron-Frobenius operator $\Lambda$

$$
\Lambda_{h}(\theta)(\phi):=\Lambda\left(e^{\theta h} \phi\right) .
$$

We collect here some properties of the family of pertubations of $\Lambda$.

Proposition 3.1. Assume that $h$ is in $W^{1,2+\epsilon}$ for some $\epsilon>0$. Then

(1) $\Lambda_{h}(0)=\Lambda$.

(2) For every $\theta \in \mathbb{C}, \Lambda_{h}(\theta)$ is a bounded linear operator on $W^{1,2}$.

(3) The maps $\theta \mapsto \Lambda_{h}(\theta)$ is analytic.

(4) For every $n \in \mathbb{N}, \theta \in \mathbb{C}$ and every $\phi$ in $W^{1,2}$, we have

$$
\Lambda_{h}^{n}(\theta)(\phi)=\Lambda^{n}\left(e^{\theta S_{n} h} \phi\right),
$$

where $S_{n} h:=\sum_{k=0}^{n-1} h \circ f^{k}$ for $n \geq 1$ and $S_{0} h:=0$.

Proof. For the proof of Parts (2) and (3) we will need the following inequality

$$
\|\phi \psi\|_{W^{1,2}} \leq C_{\epsilon}\|\phi\|_{W^{1,2}}\|\psi\|_{W^{1,2+\epsilon}}, \quad \phi \in W^{1,2}, \psi \in W^{1,2+\epsilon} .
$$

This inequality is a consequence of the following estimates where we use (2.2), (2.3) and the classical Hölder inequality

$$
\|\phi \psi\|_{L^{2}} \leq\|\phi\|_{L^{2}}\|\psi\|_{L^{\infty}} \leq C_{\epsilon}\|\phi\|_{L^{2}}\|\psi\|_{W^{1,2+\epsilon}}
$$

and

$$
\begin{aligned}
\|\mathrm{d}(\phi \psi)\|_{L^{2}} & \leq\|\psi \mathrm{d} \phi\|_{L^{2}}+\|\phi \mathrm{d} \psi\|_{L^{2}} \\
& \leq C_{\epsilon}\|\psi\|_{L^{\infty}}\|\mathrm{d} \phi\|_{L^{2}}+\|\mathrm{d} \psi\|_{L^{2+\epsilon}}\|\phi\|_{L^{\frac{2(2+\epsilon)}{\epsilon}}} \\
& \leq C_{\epsilon}\|\psi\|_{W^{1,2+\epsilon}}\|\mathrm{d} \phi\|_{L^{2}}+C_{\epsilon}\|\mathrm{d} \psi\|_{L^{2+\epsilon}}\|\phi\|_{W^{1,2}}
\end{aligned}
$$

Using (3.2) and (2.4), we get

$$
\left\|\Lambda\left(h^{n} \phi\right)\right\|_{W^{1,2}} \leq\|\Lambda\|_{W^{1,2}}\left\|h^{n} \phi\right\|_{W^{1,2}} \leq C\|\Lambda\|_{W^{1,2}}\|\phi\|_{W^{1,2}}\left(C\|h\|_{W^{1,2+\epsilon}}\right)^{n} .
$$

Consequently, the series $\sum_{n \geq 0} \frac{\theta^{n}}{n !} \Lambda\left(h^{n} \phi\right)$ converges normally in $W^{1,2}$. Its limit is equal to $\Lambda_{h}(\theta)(\phi)$, and

$$
\left\|\Lambda_{h}(\theta)(\phi)\right\|_{W^{1,2}} \leq C e^{C|\theta|\|h\|_{W^{1,2+\epsilon}}}\|\Lambda\|_{W^{1,2}}\|\phi\|_{W^{1,2}} .
$$

This gives (2) and (3).

Part (4) is proved by induction. Using (1.1), we get $\Lambda^{n}\left(\left(h \circ f^{n}\right) \phi\right)=h \Lambda^{n} \phi$ for $n \geq 0$. Hence

$$
\begin{aligned}
\Lambda^{n}\left(e^{\theta S_{n} h} \phi\right) & =\Lambda\left(\Lambda^{n-1}\left(e^{\theta h \circ f^{n-1}} e^{\theta S_{n-1} h} \phi\right)\right) \\
& =\Lambda\left(e^{\theta h} \Lambda^{n-1}\left(e^{\theta S_{n-1} h} \phi\right)\right) \\
& =\Lambda_{h}(\theta)\left(\Lambda^{n-1}\left(e^{\theta S_{n-1} h} \phi\right)\right) .
\end{aligned}
$$

This completes the proof.

The spectrum of $\Lambda_{h}(\theta)$ is described by the following pertubation theorem. 
Theorem 3.2. Let $h$ be a real-valued function in $W^{1,2+\epsilon}$. Then there exists a real number $a>0$ such that for $|\theta|<a$ and $n \in \mathbb{N}$, we have the following decomposition

$$
\Lambda_{h}^{n}(\theta)=\lambda^{n}(\theta) \Phi(\theta)+\Psi^{n}(\theta)
$$

where

- $\lambda(\theta)$ is the eigenvalue with maximal modulus of $\Lambda_{h}(\theta), \lambda(0)=1$, and $|\lambda(\theta)|>$ $\frac{2+\rho(\Psi(0))}{3}=: \rho_{1}<1$;

- $\Phi(\theta)$ is the projection of $W^{1,2}$ onto the eigenspace $W^{1,2}(\lambda(\theta))$ which is of dimension 1 , and $\Phi(0)(\phi)=\mu(\phi)$;

- $\Psi(\theta)$ is a bounded linear operator on $W^{1,2}$ with $\rho(\Psi(\theta))<\rho_{1}$, and

$$
\Psi(\theta) \circ \Phi(\theta)=\Phi(\theta) \circ \Psi(\theta)=0 .
$$

- $\theta \mapsto \Phi(\theta), \theta \mapsto \Psi(\theta), \theta \mapsto \lambda(\theta)$ are analytic maps on the disc $\{|\theta|<a\}$.

Proof. Observe that $\Lambda(1)=1$. Then, constant functions are eigenfunctions associated to the eigenvalue 1 of the operator $\Lambda_{h}(0)=\Lambda$. If $\lambda(0), \Phi(0)$ are as above and if $\Psi(0):=\Lambda-\Phi(0)$, then

$$
\Lambda_{h}(0)=\Lambda=\lambda(0) \Phi(0)+\Psi(0)
$$

It follows from (2.6) that $\rho(\Psi(0)) \leq \frac{1}{\sqrt{d}}<1$. Hence, $\lambda(0)=1$ is the unique eigenvalue of maximal modulus of $\Lambda$ and $W^{1,2}(\lambda(0))$ is of dimension 1. Using Proposition 3.1. it is enough to apply the Rellich pertubation method described in [10, Chapter VII] (see also [3, Proposition 5.2], [17).

Now we are in position to prove the first main result.

Theorem A. Let $f: \mathbb{P}^{1} \rightarrow \mathbb{P}^{1}$ be a rational map of degree $d \geq 2$. Let $g$ be $a$ real-valued function in $W^{1,2}$ and $h$ a real-valued function in $W^{1,2+\epsilon}, \epsilon>0$, such that $\mu(g)=1, \mu(h)=0$ and $g(z) \geq 0$ for $\mu$-a.e. $z \in \mathbb{P}^{1}$. Assume $h$ is not a coboundary and let $\sigma$ be the positive number defined by

$$
\sigma^{2}:=-\int_{\mathbb{P}^{1}} h^{2} \mathrm{~d} \mu+2 \sum_{n \geq 0} \int_{\mathbb{P}^{1}} h\left(h \circ f^{n}\right) \mathrm{d} \mu=\lim _{n \rightarrow \infty} \int_{\mathbb{P}^{1}}\left(\frac{1}{\sqrt{n}} S_{n} h\right)^{2} \mathrm{~d} \mu .
$$

Define the probability measure

$$
\nu:=g \mu
$$

Then for every real number $v$, we have

$$
\lim _{n \rightarrow \infty} \nu\left\{\frac{S_{n} h}{\sigma \sqrt{n}} \leq v\right\}=\frac{1}{\sqrt{2 \pi}} \int_{-\infty}^{v} e^{-\frac{t^{2}}{2}} \mathrm{~d} t
$$

When $g=1$, we obtain the CLT for $\mu$. Since the theorem holds for every $\nu=g \mu$, the set $\left\{S_{n} h \leq \sigma \sqrt{n} v\right\}$ is asymptotically equidistributed with respect to $\mu$ as $n \rightarrow \infty$, because this set is independent of $g$. 
Proof. Using Proposition 3.1 and Theorem 3.2 the proof follows the same lines as in [3. Théorème 6.8] or [23, Théorème 2]. For the sake of clarity, we give here the main aguments. The proof is divided into four steps.

Step 1: Proof of the equality $\lambda^{\prime}(0)=0$. Since $\mu$ is totally invariant, we have $\int \Lambda^{n}(\phi) \mathrm{d} \mu=\int \phi \mathrm{d} \mu$ for every $\phi \in L^{1}(\mu)$. This, Proposition 3.1 and Theorem 3.2 imply that for $\left|\frac{t}{n}\right|<a$,

$$
\begin{aligned}
\int_{\mathbb{P}^{1}} e^{\frac{i t S_{n} h}{n}} \mathrm{~d} \mu & =\int_{\mathbb{P}^{1}} \Lambda^{n}\left(e^{\frac{i t S_{n} h}{n}}\right) \mathrm{d} \mu=\int_{\mathbb{P}^{1}} \Lambda_{h}^{n}\left(\frac{i t}{n}\right)(1) \mathrm{d} \mu \\
& =\lambda^{n}\left(\frac{i t}{n}\right) \int_{\mathbb{P}^{1}} \Phi\left(\frac{i t}{n}\right)(1) \mathrm{d} \mu+\int_{\mathbb{P}^{1}} \Psi^{n}\left(\frac{i t}{n}\right)(1) \mathrm{d} \mu .
\end{aligned}
$$

We then use the Taylor expansion of order 2 at 0 of $\lambda\left(\frac{i t}{n}\right)$ and $\Phi\left(\frac{i t}{n}\right)$. Moreover, we use the estimate $\left\|\Psi^{n}\left(\frac{i t}{n}\right)(\phi)\right\|_{W^{1,2}} \leq C \rho_{1}^{n}$. Therefore, one obtains that

$$
\lim _{n \rightarrow \infty} \int_{\mathbb{P}^{1}} e^{i t \frac{S_{n} h}{n}} \mathrm{~d} \mu=e^{i t \lambda^{\prime}(0)}
$$

By Birkhoff's theorem, $\lim _{n \rightarrow \infty} \frac{S_{n} h}{n}=\mu(h)=0, \mu$-a.e. Hence, $\lambda^{\prime}(0)=0$.

Step 2: Proof of the equality $\lambda^{\prime \prime}(0)=\sigma^{2}$. Equality (3.3) is easy to check using the invariance of $\mu$. On the other hand,

$$
\int_{\mathbb{P}^{1}}\left(\frac{S_{n} h}{\sqrt{n}}\right)^{2} \mathrm{~d} \mu=-\frac{\partial^{2}}{\partial t^{2}}\left[\int_{\mathbb{P}^{1}} e^{\frac{i t}{\sqrt{n}} S_{n} h} \mathrm{~d} \mu\right]_{t=0} .
$$

By Theorem 3.2, we may rewrite the expression in brackets in (3.4) as follows

$$
\begin{aligned}
\int_{\mathbb{P}^{1}} e^{\frac{i t}{\sqrt{n}} S_{n} h} \mathrm{~d} \mu & =\int_{\mathbb{P}^{1}} \Lambda_{h}^{n}\left(\frac{i t}{\sqrt{n}}\right)(1) \mathrm{d} \mu \\
& =\lambda^{n}\left(\frac{i t}{\sqrt{n}}\right) \int_{\mathbb{P}^{1}} \Phi\left(\frac{i t}{\sqrt{n}}\right)(1) \mathrm{d} \mu+\int_{\mathbb{P}^{1}} \Psi^{n}\left(\frac{i t}{\sqrt{n}}\right)(1) \mathrm{d} \mu .
\end{aligned}
$$

The derivative of the second term can be bounded by $C \rho_{1}^{n}$ using Cauchy's formula. We use the Taylor expansion of order 2 at 0 of $\lambda\left(\frac{i t}{\sqrt{n}}\right)$ and $\Phi\left(\frac{i t}{\sqrt{n}}\right)$ and insert them into the above expansion. After taking the second derivative we see that the right hand side of (3.4) is equal to $\lambda^{\prime \prime}(0)$. We use here the equality $\lambda^{\prime}(0)=0$.

Step 3: Proof of the equality $\lim _{n \rightarrow \infty} \int \Lambda_{h}^{n}\left(\frac{i t}{\sqrt{n}}\right)(g) \mathrm{d} \mu=e^{-\frac{\sigma^{2} t^{2}}{2}}$. By Theorem 3.2 we have

$$
\int_{\mathbb{P}^{1}} \Lambda_{h}^{n}\left(\frac{i t}{\sqrt{n}}\right)(g) \mathrm{d} \mu=\lambda^{n}\left(\frac{i t}{\sqrt{n}}\right) \int_{\mathbb{P}^{1}} \Phi\left(\frac{i t}{\sqrt{n}}\right)(g) \mathrm{d} \mu+\int_{\mathbb{P}^{1}} \Psi^{n}\left(\frac{i t}{\sqrt{n}}\right)(g) \mathrm{d} \mu .
$$


As in Step 1, the last term in the above expression tends to 0 as $n \rightarrow \infty$. On the other hand, using the previous steps and the hypothesis $\mu(g)=1$, a straightforward computation gives that

$$
\lambda^{n}\left(\frac{i t}{\sqrt{n}}\right)=e^{-\frac{\sigma^{2} t^{2}}{2}\left[1+O\left(\frac{t}{\sqrt{n}}\right)\right]} \text { and } \int_{\mathbb{P}^{1}} \Phi\left(\frac{i t}{\sqrt{n}}\right)(g) \mathrm{d} \mu=1+O\left(\frac{t}{\sqrt{n}}\right)
$$

Replacing them in the right hand side of (3.5), we obtain the desired equality.

Step 4: Conclusion. Using Proposition 3.1(4), we may rephrase the result of Step 3 as $\lim _{n \rightarrow \infty} \int e^{i t \frac{S_{n} h}{\sigma \sqrt{n}}} d \nu=e^{-\frac{t^{2}}{2}}$. Since the right hand side of this equality is the Fourier transform of the normal law $\mathcal{N}(0,1)$, the Paul Lévy's method of characteristic functions implies that the sequence of random variables $\left(\frac{S_{n} h}{\sigma \sqrt{n}}\right)_{n=1}^{\infty}$ converges in law to the normal law. This implies the result.

We come to the second main result.

Theorem B. Under the hypothesis of Theorem A, there is a constant $C$ such that

$$
\sup _{v \in \mathbb{R}}\left|\nu\left\{\frac{S_{n} h}{\sigma \sqrt{n}} \leq v\right\}-\frac{1}{\sqrt{2 \pi}} \int_{-\infty}^{v} e^{-\frac{t^{2}}{2}} \mathrm{~d} t\right| \leq \frac{C}{\sqrt{n}}
$$

Proof. First we recall the Berry-Essen inequality (see [11]) in our context. Namely, there is a positive constant $K$ such that for $V>0$,

$$
\sup _{v \in \mathbb{R}}\left|\nu\left\{\frac{S_{n} h}{\sigma \sqrt{n}} \leq v\right\}-\frac{1}{\sqrt{2 \pi}} \int_{-\infty}^{v} e^{\frac{-t^{2}}{2}} \mathrm{~d} t\right| \leq \frac{K}{V}+\frac{1}{\pi} \int_{-V}^{V} \frac{1}{|t|}\left|\int_{\mathbb{P}^{1}} e^{i t \frac{S_{n} h}{\sigma \sqrt{n}}} \mathrm{~d} \nu-e^{-\frac{t^{2}}{2}}\right| \mathrm{d} t .
$$

Choose $V=\sigma \sqrt{n} a^{\prime}$ with $a^{\prime}<a$ small enough, where $a$ is the constant given by Theorem 3.2. Then for every $t \in[-V, V]$, we have $\left|\frac{t}{\sigma \sqrt{n}}\right| \leq a^{\prime}<a$. Consequently, applying Proposition [3.1(4) and Theorem 3.2 yields that

$$
\begin{aligned}
\int_{\mathbb{P}^{1}} e^{i t \frac{S_{n} h}{\sigma \sqrt{n}}} \mathrm{~d} \nu & =\int_{\mathbb{P}^{1}} e^{i t \frac{S_{n} h}{\sigma \sqrt{n}}} g \mathrm{~d} \mu=\int_{\mathbb{P}^{1}} \Lambda_{h}^{n}\left(\frac{i t}{\sigma \sqrt{n}}\right)(g) \mathrm{d} \mu \\
& =\lambda^{n}\left(\frac{i t}{\sigma \sqrt{n}}\right) \int_{\mathbb{P}^{1}} \Phi\left(\frac{i t}{\sigma \sqrt{n}}\right)(g) \mathrm{d} \mu+\int_{\mathbb{P}^{1}} \Psi^{n}\left(\frac{i t}{\sigma \sqrt{n}}\right)(g) \mathrm{d} \mu .
\end{aligned}
$$

Theorem 3.2 also implies that $\int \Psi(0)(\phi) \mathrm{d} \mu=0$ for every $\phi$. Moreover, the $W^{1,2}$ norm of the operator $\Psi(\theta)-\Psi(0)$ satisfies $\|\Psi(\theta)-\Psi(0)\|_{W^{1,2}} \leq C|\theta|$. Hence we can apply this to $\phi:=\Psi^{n-1}\left(\frac{i t}{\sigma \sqrt{n}}\right)(g)$ and obtain

$$
\left|\int_{\mathbb{P}^{1}} \Psi^{n}\left(\frac{i t}{\sigma \sqrt{n}}\right)(g) \mathrm{d} \mu\right|=\left|\int_{\mathbb{P}^{1}}\left[\Psi\left(\frac{i t}{\sigma \sqrt{n}}\right)-\Psi(0)\right] \circ \Psi^{n-1}\left(\frac{i t}{\sigma \sqrt{n}}\right)(g) \mathrm{d} \mu\right| \leq \frac{C \rho_{1}^{n-1}|t|}{\sqrt{n}} .
$$


Now, we use the computation in the third step of the proof of Theorem A. From the inequalities $1-e^{-s x} \leq e^{s x}-1 \leq s e^{x}$ for $0 \leq s \leq 1$ and $x \geq 0$, we deduce that

$$
\left|\lambda^{n}\left(\frac{i t}{\sigma \sqrt{n}}\right)-e^{-\frac{t^{2}}{2}}\right| \leq e^{-\frac{t^{2}}{2}}\left|e^{O\left(\frac{t^{3}}{\sigma \sqrt{n}}\right)}-1\right| \leq e^{-\frac{t^{2}}{2}}\left(e^{\frac{t^{2}}{4} \frac{C|t|}{\sigma \sqrt{n}}}-1\right) \leq \frac{C e^{-\frac{t^{2}}{4}}|t|}{\sigma \sqrt{n}}
$$

since $\frac{C|t|}{\sigma \sqrt{n}} \leq C a^{\prime} \leq 1$ when $a^{\prime}$ is small enough. It follows that

$$
\left|\lambda^{n}\left(\frac{i t}{\sigma \sqrt{n}}\right) \int_{\mathbb{P}^{1}} \Phi\left(\frac{i t}{\sigma \sqrt{n}}\right)(g) \mathrm{d} \mu-e^{-\frac{t^{2}}{2}}\right| \leq \frac{C e^{-\frac{t^{2}}{4}}|t|}{\sqrt{n}} .
$$

Hence,

$$
\int_{-V}^{V} \frac{1}{|t|}\left|\int_{\mathbb{P}^{1}} e^{i t \frac{S_{n} h}{\sigma \sqrt{n}}} \mathrm{~d} \nu-e^{-\frac{t^{2}}{2}}\right| \mathrm{d} t \leq \frac{C}{\sqrt{n}} \int_{-V}^{V}\left(e^{-\frac{t^{2}}{4}}+\rho_{1}^{n-1}\right) \mathrm{d} t \leq \frac{C^{\prime}\left(1+V \rho_{1}^{n-1}\right)}{\sqrt{n}} .
$$

Now we substitute $V=\sigma \sqrt{n} a^{\prime}$ into the latter estimate. Consequently, it follows from the Berry-Essen inequality that

$$
\sup _{v \in \mathbb{R}}\left|\nu\left\{\frac{S_{n} h}{\sigma \sqrt{n}} \leq v\right\}-\frac{1}{\sqrt{2 \pi}} \int_{-\infty}^{v} e^{\frac{-t^{2}}{2}} \mathrm{~d} t\right| \leq \frac{K}{\sqrt{n} \sigma a^{\prime}}+\frac{1}{\pi} \frac{C^{\prime}\left(1+\sqrt{n} \sigma a^{\prime} \rho_{1}^{n-1}\right)}{\sqrt{n}} \leq \frac{C^{\prime \prime}}{\sqrt{n}} .
$$

This completes the proof of the theorem.

\section{Spectral Decomposition of $\Lambda_{h}(i t)$ For $t$ ReAL}

We begin with a version of the classical Ionescu-Tulcea and Marinescu theorem (see [16, 21]).

Theorem 4.1. Let $\mathcal{V}, \mathcal{L}$ and $\mathcal{K}$ be three Banach spaces respectively endowed with norms $\|\cdot\|_{\mathcal{V}},\|\cdot\|_{\mathcal{L}},\|\cdot\|_{\mathcal{K}}$. Suppose that $\mathcal{V} \subset \mathcal{L} \subset \mathcal{K}$ and that there is a positive constant $C$ satisfying the following properties

(a) If $\left(\phi_{n}\right)_{n=1}^{\infty} \subset \mathcal{V}$ with $\left\|\phi_{n}\right\|_{\mathcal{V}} \leq 1$, then there is a subsequence $\left(\phi_{n_{j}}\right)_{j=1}^{\infty}$ and an element $\phi \in \mathcal{V}$ such that $\lim _{j \rightarrow \infty}\left\|\phi_{n_{j}}-\phi\right\|_{\mathcal{L}}=0$ and $\|\phi\|_{\mathcal{V}} \leq C$.

(a) ${ }^{\prime}\|\phi\|_{\mathcal{K}} \leq C\|\phi\|_{\mathcal{L}}, \phi \in \mathcal{L}$.

Let $T: \mathcal{K} \rightarrow \mathcal{K}$ be a bounded linear operator such that $T(\mathcal{V}) \subset \mathcal{V}$. Assume that

(b) (Doeblin-Fortet inequality) There are two positive constants $\alpha<1$ and $\beta$ such that $\|T(\phi)\|_{\mathcal{V}} \leq \alpha\|\phi\|_{\mathcal{V}}+\beta\|\phi\|_{\mathcal{L}}, \phi \in \mathcal{V}$.

(c) $\rho\left(\left.T\right|_{\mathcal{V}}\right) \leq 1$.

(d) $\left.T\right|_{\mathcal{V}}$ admits no eigenvalue of modulus 1 , that is, if $\phi \in \mathcal{V} \backslash\{0\}$ and $\lambda \in \mathbb{C}$ satisfy $T(\phi)=\lambda \phi$, then $|\lambda| \neq 1$.

Then $\rho\left(\left.T\right|_{\mathcal{V}}\right)<1$.

Proof. It suffices to prove for $|\lambda|=1$ that $(I-\lambda T)$ admits a bounded linear inverse from $\mathcal{V}$ onto $\mathcal{V}$. Fix a $\lambda_{0} \in \mathbb{C}$ such that $\left|\lambda_{0}\right|=1$ and an arbitrary $\psi \in \mathcal{V}$. We would like to find a suitable $\phi \in \mathcal{V}$ such that $\left(I-\lambda_{0} T\right) \phi=\psi$. To this end pick an arbitrary sequence $\left(\lambda_{n}\right)_{n=1}^{\infty} \subset \mathbb{C},\left|\lambda_{n}\right|<1$, converging to $\lambda_{0}$. 
Observe that by (c), for every $\lambda \in \mathbb{C}$ with $|\lambda|<1, \rho(\lambda T)=|\lambda| \rho(T)<1$. Therefore, the series $(I-\lambda T)^{-1}:=\sum_{n \geq 0} \lambda^{n} T^{n}$ defines a bounded linear operator from $\mathcal{V}$ onto $\mathcal{V}$ which is the inverse of $(I-\lambda T)$. Consequently, for every $n \geq 1$, one may find $\phi_{n} \in \mathcal{V}$ such that

$$
\left(I-\lambda_{n} T\right) \phi_{n}=\psi
$$

We claim that

$$
M:=\sup _{n \geq 1}\left\|\phi_{n}\right\|_{\mathcal{L}}<\infty .
$$

If not, there is a subsequence $\left(\phi_{n_{j}}\right)_{j=1}^{\infty}$ such that $\lim _{j \rightarrow \infty}\left\|\phi_{n_{j}}\right\|_{\mathcal{L}}=\infty$. To simplify the notation assume that $n_{j}=j$. Put $\phi_{j}^{\prime}:=\frac{\phi_{j}}{\left\|\phi_{j}\right\|_{\mathcal{L}}}$. Then (4.1) may be rewritten as

$$
\phi_{j}^{\prime}=\lambda_{j} T \phi_{j}^{\prime}+\frac{\psi}{\left\|\phi_{j}\right\|_{\mathcal{L}}} .
$$

Consequently, using (b), we get

$$
\left\|\phi_{j}^{\prime}\right\|_{\mathcal{V}} \leq\left\|T \phi_{j}^{\prime}\right\|_{\mathcal{V}}+\frac{\|\psi\|_{\mathcal{V}}}{\left\|\phi_{j}\right\|_{\mathcal{L}}} \leq \alpha\left\|\phi_{j}^{\prime}\right\|_{\mathcal{V}}+\beta+\frac{\|\psi\|_{\mathcal{V}}}{\left\|\phi_{j}\right\|_{\mathcal{L}}} .
$$

Hence,

$$
\left\|\phi_{j}^{\prime}\right\|_{\mathcal{V}} \leq \frac{\beta+\frac{\|\psi\|_{\mathcal{V}}}{\left\|\phi_{j}\right\|_{\mathcal{L}}}}{1-\alpha}
$$

Since by assumption $\lim _{j \rightarrow \infty}\left\|\phi_{j}\right\|_{\mathcal{L}}=\infty$, it follows from the latter estimate that $\left(\phi_{j}^{\prime}\right)_{j=1}^{\infty}$ is a bounded sequence in $\mathcal{V}$. Therefore, by (a), there is a subsequence of $\left(\phi_{j}^{\prime}\right)_{j=1}^{\infty}$ which converges in $\mathcal{L}$ to an element $\phi^{\prime} \in \mathcal{V}$. Consequently, letting $j$ tend to $\infty$ in (4.3) and using (a)' and the continuity of $T: \mathcal{K} \rightarrow \mathcal{K}$, we obtain

$$
\phi^{\prime}=\lambda_{0} T \phi^{\prime} \quad \text { and } \quad\left\|\phi^{\prime}\right\|_{\mathcal{L}}=1,
$$

which contradicts (d). Hence, the proof of (4.2) is complete.

Using (4.2) and arguing as in (4.4)-(4.5), we can show that

$$
\left\|\phi_{n}\right\|_{\mathcal{V}} \leq \frac{M \beta+\|\psi\|_{\mathcal{V}}}{1-\alpha}
$$

By (a), there is a subsequence of $\left(\phi_{n}\right)_{n=1}^{\infty}$ which converges in $\mathcal{L}$ to an element $\phi \in \mathcal{V}$. This, combined with (4.1), implies that $\left(I-\lambda_{0} T\right) \phi=\psi$. Hence, $\left(I-\lambda_{0} T\right)$ is onto $\mathcal{V}$. Recall from $(\mathrm{c})-(\mathrm{d})$ that $\left(I-\lambda_{0} T\right)$ is one-to-one. If $\left(\psi^{(n)}\right) \subset \mathcal{V}$ and $\left(\phi^{(n)}\right) \subset \mathcal{V}$ satisfy $\left\|\psi^{(n)}\right\|_{\mathcal{V}}=1$ and $\left(I-\lambda_{0} T\right) \phi^{(n)}=\psi^{(n)}$, arguing as in (4.4)-(4.6) we show that $\left(\phi^{(n)}\right)$ is bounded in $\mathcal{L}$, and then in $\mathcal{V}$. Therefore, $\left(I-\lambda_{0} T\right)^{-1}$ exists and is bounded. This completes the proof.

The remaining of this section is devoted to an application of Theorem 4.1 in the case $\mathcal{K}:=L^{1}, \mathcal{L}:=L^{\frac{4+2 \epsilon}{\epsilon}}$ and $\mathcal{V}:=W^{1,2}$.

Proposition 4.2. Let $h$ be a real-valued function in $W^{1,2+\epsilon}, \epsilon>0$. Then, the hypotheses (a)-(b) of Theorem [4.1] are fulfilled with $\mathcal{K}:=L^{1}, \mathcal{L}:=L^{\frac{4+2 \epsilon}{\epsilon}}, \mathcal{V}:=W^{1,2}$ and $T:=\Lambda_{h}(i t), t \in \mathbb{R}$. 
Proof. The hypothesis (a) is a consequence of the Sobolev embedding theorem. The hypothesis (a)' is obvious. The operator $\Lambda_{h}(i t): \mathcal{K} \rightarrow \mathcal{K}$ is bounded since for every $\phi \in L^{1}$, we have

$$
\begin{aligned}
\left\|\Lambda_{h}(i t)(\phi)\right\|_{L^{1}} & =\left\|\Lambda\left(e^{i t h} \phi\right)\right\|_{L^{1}} \leq\|\Lambda(|\phi|)\|_{L^{1}} \\
& =\int_{\mathbb{P}^{1}} \Lambda(|\phi|) \omega=\frac{1}{d} \int_{\mathbb{P}^{1}}|\phi| f^{*}(\omega) \leq C\|\phi\|_{L^{1}} .
\end{aligned}
$$

We now check condition (b). Let $\phi \in W^{1,2}$. We first estimate $\left\|\mathrm{d} \Lambda_{h}(i t)(\phi)\right\|_{L^{2}}$. To this end we repeatedly apply the Cauchy-Schwarz inequality. This inequality implies, in particular, that $i f_{*}(\alpha) \wedge f_{*}(\bar{\alpha}) \leq d f_{*}(i \alpha \wedge \bar{\alpha})$ or equivalently $i \Lambda(\alpha) \wedge \Lambda(\bar{\alpha}) \leq$ $\Lambda(i \alpha \wedge \bar{\alpha})$, where $\alpha$ is a $(1,0)$-form. Recall that $\mathrm{d}=\partial+\bar{\partial}$. Using Proposition $[3.1(4)$ we obtain

$$
\begin{aligned}
& i \partial \Lambda_{h}(i t)(\phi) \wedge \overline{\partial \Lambda_{h}(i t)(\phi)} \\
&=\frac{i}{d^{2}} f_{*}\left(\partial\left(e^{i t h} \phi\right)\right) \wedge f_{*}\left(\bar{\partial}\left(e^{-i t h} \bar{\phi}\right)\right) \\
& \leq \frac{1}{d} f_{*}\left(i \partial\left(e^{i t h} \phi\right) \wedge \bar{\partial}\left(e^{-i t h} \bar{\phi}\right)\right) \\
&=\frac{1}{d} f_{*}(i \partial \phi \wedge \overline{\partial \phi})+\frac{1}{d} f_{*}\left(i \phi e^{-i t h} \partial e^{i t h} \wedge \overline{\partial \phi}\right) \\
& \quad+\frac{1}{d} f_{*}\left(i \bar{\phi} e^{i t h} \partial \phi \wedge \bar{\partial} e^{-i t h}\right)+\frac{1}{d} f_{*}\left(i|\phi|^{2} \partial e^{i t h} \wedge \bar{\partial} e^{-i t h}\right) \\
& \leq \frac{11}{9 d} f_{*}(i \partial \phi \wedge \overline{\partial \phi})+\frac{19}{d} f_{*}\left(i|\phi|^{2} \partial e^{i t h} \wedge \bar{\partial} e^{-i t h}\right) .
\end{aligned}
$$

In the last line, we use the inequality $a b \leq \frac{1}{9} a^{2}+9 b^{2}$. Consequently, since $\|\mathrm{d} \psi\|_{L^{2}}=$ $2\|\partial \psi\|_{L^{2}}$ for every function $\psi$ and $\int_{\mathbb{P}^{1}} f_{*}(\beta)=\int_{\mathbb{P}^{1}} \beta$ for every 2 -form $\beta$, we have

$$
\begin{aligned}
\left\|\mathrm{d} \Lambda_{h}(i t)(\phi)\right\|_{L^{2}}^{2} & =2 \int_{\mathbb{P}^{1}} i \partial \Lambda_{h}(i t)(\phi) \wedge \overline{\partial \Lambda_{h}(i t)(\phi)} \\
& \leq \frac{22}{9 d} \int_{\mathbb{P}^{1}} f_{*}(i \partial \phi \wedge \overline{\partial \phi})+\frac{38}{d} \int_{\mathbb{P}^{1}}\left(f^{n}\right)_{*}\left(i|\phi|^{2} \partial e^{i t h} \wedge \bar{\partial} e^{-i t h}\right) \\
& \leq \frac{11}{18}\|\mathrm{~d} \phi\|_{L^{2}}^{2}+19 \int_{\mathbb{P}^{1}} i|\phi|^{2} \partial e^{i t h} \wedge \bar{\partial} e^{-i t h} .
\end{aligned}
$$

Applying Cauchy-Schwarz inequality and Hölder's inequality, we may estimate the last integral

$$
\int_{\mathbb{P}^{1}} i|\phi|^{2} \partial e^{i t h} \wedge \bar{\partial} e^{-i t h}=|t|^{2} \int_{\mathbb{P}^{1}} i|\phi|^{2} \partial h \wedge \bar{\partial} h \leq|t|^{2}\|\phi\|_{L^{\frac{4+2 \epsilon}{\epsilon}}}^{2}\|\mathrm{~d} h\|_{L^{2+\epsilon}}^{2} .
$$

Finally, we have shown that

$$
\left\|\mathrm{d} \Lambda_{h}(i t)(\phi)\right\|_{L^{2}} \leq \sqrt{\frac{11}{18}}\|\mathrm{~d} \phi\|_{L^{2}}+C(h, \epsilon, t)\|\phi\|_{L^{\frac{4+2 \epsilon}{\epsilon}}} .
$$


On the other hand, we deduce from (2.2) and (2.5)-(2.6) that $\left\|\Lambda_{h}(i t)(\phi)\right\|_{L^{2}} \leq\|\Lambda(|\phi|)\|_{L^{2}} \leq \int \Lambda\left(|\phi|^{2}\right) \omega=\frac{1}{d} \int|\phi|^{2} f^{*}(\omega) \leq C\|\phi\|_{L^{2}} \leq C^{\prime}\|\phi\|_{L^{\frac{4+2 \epsilon}{\epsilon}}}$.

This, combined with (4.8), implies the Doeblin-Fortet inequality with $\alpha:=\sqrt{\frac{11}{18}}$, and $\beta:=C^{\prime}+C(h, \epsilon, t)$.

Proposition 4.3. Under the hypothesis of Proposition [4.2, the hypothesis (c) in Theorem 4.1 holds, i.e. we have $\rho\left(\left.\Lambda_{h}(i t)\right|_{W^{1,2}}\right) \leq 1$ for every $t \in \mathbb{R}$.

Proof. First, using Proposition 3.1(4) and (2.2)-(2.6), we have

$$
\begin{aligned}
\left\|\Lambda_{h}^{n}(i t)(\phi)\right\|_{L^{2}} & =\left\|\Lambda^{n}\left(e^{i t S_{n} h} \phi\right)\right\|_{L^{2}} \leq\left\|\Lambda^{n}(|\phi|)\right\|_{L^{2}} \\
& \leq\left\|\Lambda^{n}(|\phi|)\right\|_{W^{1,2}} \leq C\||\phi|\|_{W^{1,2}} \leq C^{\prime}\|\phi\|_{W^{1,2}} .
\end{aligned}
$$

Now, we use Proposition 3.1(4) and the Cauchy-Schwarz inequality repeatedly to estimate $\left\|\mathrm{d} \Lambda_{h}^{n}(i t)(\phi)\right\|_{L^{2}}$. As in Proposition 4.2, we obtain

$$
\begin{aligned}
& i \partial \Lambda_{h}^{n}(i t)(\phi) \wedge \overline{\partial \Lambda_{h}^{n}(i t)(\phi)} \\
&=i \Lambda^{n}\left(\partial\left(e^{i t S_{n} h} \phi\right)\right) \wedge \Lambda^{n}\left(\bar{\partial}\left(e^{-i t S_{n} h} \bar{\phi}\right)\right) \\
& \leq \quad i \Lambda^{n}\left(i t e^{i t S_{n} h} \phi \partial S_{n} h+e^{i t S_{n} h} \partial \phi\right) \wedge \Lambda^{n}\left(-i t e^{-i t S_{n} h} \bar{\phi} \bar{\partial} S_{n} h+e^{-i t S_{n} h} \overline{\partial \phi}\right) \\
& \leq 2 i \Lambda^{n}\left(e^{i t S_{n} h} \partial \phi\right) \wedge \Lambda^{n}\left(e^{-i t S_{n} h} \overline{\partial \phi}\right) \\
&+2 t^{2} i \Lambda^{n}\left(e^{i t S_{n} h} \phi \partial S_{n} h\right) \wedge \Lambda^{n}\left(e^{-i t S_{n} h} \bar{\phi} \bar{\partial} S_{n} h\right) \\
&(4.10) \equiv I+2 t^{2} I I .
\end{aligned}
$$

For the first term we have

$$
I \leq 2 \Lambda^{n}(i \partial \phi \wedge \overline{\partial \phi})
$$

For the second one we have

$$
\begin{aligned}
I I & =i \Lambda^{n}\left(e^{i t S_{n} h} \phi \sum_{k=0}^{n-1} \partial\left(h \circ f^{k}\right)\right) \wedge \Lambda^{n}\left(e^{-i t S_{n} h} \bar{\phi} \sum_{k=0}^{n-1} \bar{\partial}\left(h \circ f^{k}\right)\right) \\
& \leq n \sum_{k=0}^{n-1} i \Lambda^{n}\left(e^{i t S_{n} h} \phi \partial\left(h \circ f^{k}\right)\right) \wedge \Lambda^{n}\left(e^{-i t S_{n} h} \bar{\phi} \bar{\partial}\left(h \circ f^{k}\right)\right) \\
& =n \sum_{k=0}^{n-1} i \Lambda^{n-k}\left(\Lambda^{k}\left(e^{i t S_{n} h} \phi \partial\left(h \circ f^{k}\right)\right)\right) \wedge \Lambda^{n-k}\left(\Lambda^{k}\left(e^{-i t S_{n} h} \bar{\phi} \bar{\partial}\left(h \circ f^{k}\right)\right)\right) \\
& \leq n \sum_{k=0}^{n-1} \Lambda^{n-k}\left(i \Lambda^{k}\left(e^{i t S_{n} h} \phi \partial\left(h \circ f^{k}\right)\right) \wedge \Lambda^{k}\left(e^{-i t S_{n} h} \bar{\phi} \bar{\partial}\left(h \circ f^{k}\right)\right)\right) .
\end{aligned}
$$

Observe that for any complex-valued function $\psi$, we have $i \Lambda^{k}\left(\psi \partial\left(h \circ f^{k}\right)\right) \wedge \Lambda^{k}\left(\psi \bar{\partial}\left(h \circ f^{k}\right)\right)=i \Lambda^{k}(\psi) \partial h \wedge \Lambda^{k}(\psi) \bar{\partial} h \leq i \Lambda^{k}(|\psi|)^{2} \partial h \wedge \bar{\partial} h$. 
Then

$$
I I \leq n \sum_{k=0}^{n-1} \Lambda^{n-k}\left(i \Lambda^{k}(|\phi|)^{2} \partial h \wedge \bar{\partial} h\right) .
$$

This, combined with (4.10)- (4.11), implies that

$$
\begin{aligned}
\left\|\mathrm{d} \Lambda_{h}^{n}(i t)(\phi)\right\|_{L^{2}}^{2} \leq & \frac{4}{d^{n}} \int_{\mathbb{P}^{1}}\left(f^{n}\right)_{*}(i \partial \phi \wedge \overline{\partial \phi}) \\
& +4 n t^{2} \sum_{k=0}^{n-1} \frac{1}{d^{n-k}} \int_{\mathbb{P}^{1}}\left(f^{n-k}\right)_{*}\left(i \Lambda^{k}(|\phi|)^{2} \partial h \wedge \bar{\partial} h\right) \\
= & \frac{4}{d^{n}} \int_{\mathbb{P}^{1}} i \partial \phi \wedge \overline{\partial \phi}+4 n t^{2} \sum_{k=0}^{n-1} \frac{1}{d^{n-k}} \int_{\mathbb{P}^{1}} i \Lambda^{k}(|\phi|)^{2} \partial h \wedge \bar{\partial} h .
\end{aligned}
$$

Applying Hölder's inequality, (2.2) and (4.9) yields that

$$
\int_{\mathbb{P}^{1}} i \Lambda^{k}(|\phi|)^{2} \partial h \wedge \bar{\partial} h \leq\left\|\Lambda^{k}(|\phi|)\right\|_{L^{\frac{4+2 \epsilon}{\epsilon}}}^{2}\|\mathrm{~d} h\|_{L^{2+\epsilon}}^{2} \leq C_{\epsilon}\|\phi\|_{W^{1,2}}^{2}\|\mathrm{~d} h\|_{L^{2+\epsilon}}^{2} .
$$

Since $\sum \frac{1}{d^{n-k}} \leq 2$, it follows that

$$
\left\|\mathrm{d} \Lambda_{h}^{n}(i t)(\phi)\right\|_{L^{2}} \leq\left(\sqrt{\frac{4}{d^{n}}}+\sqrt{8 n t^{2} C_{\epsilon}}\|\mathrm{d} h\|_{L^{2+\epsilon}}\right)\|\phi\|_{W^{1,2}} .
$$

Combining this and (4.9), the desired conclusion follows.

\section{Local Central Limit Theorem}

First we introduce the following notion.

Definition 5.1. A real-valued function $h$ is a multiplicative $W^{1,2}$-cocycle if there are $t>0, s \in \mathbb{R}$ and $\phi \in W^{1,2}$, $\phi$ non zero in $L^{1}(\mu)$, such that $e^{i t h(z)} \phi(z)=e^{i s} \phi(f(z))$

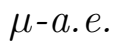

We have the following proposition.

Proposition 5.2. Let $h$ be a real-valued function in $W^{1,2+\epsilon}$. Then $h$ is not a multiplicative $W^{1,2}$-cocycle if and only if the spectral radius of $\Lambda_{h}(i t): W^{1,2} \rightarrow W^{1,2}$ is strictly smaller than 1 for every $t>0$.

Proof. First assume that $h$ is not a multiplicative $W^{1,2}$-cocycle. Suppose in order to get a contradiction that there is $t>0$ such that $\rho\left(\left.\Lambda_{h}(i t)\right|_{W^{1,2}}\right) \geq 1$. By Propositions 4.2, 4.3 and Theorem 4.1, there are $\lambda \in \mathbb{C}$ and $\phi \in W^{1,2} \backslash\{0\}$ such that $\Lambda_{h}(i t)(\phi)=$ $\lambda \phi$ and $|\lambda|=1$. It follows that $\Lambda\left(e^{i t h} \phi\right)=\lambda \phi$, then $\Lambda(|\phi|) \geq|\phi|$. Since $\mu(\Lambda(|\phi|))=$ $\mu(|\phi|)$, we deduce that $\Lambda(|\phi|)=|\phi| \mu$-a.e. We also obtain $|\phi|=\Lambda^{n}(|\phi|)$, and since $\Lambda^{n}(|\phi|)$ converges in $L^{1}(\mu)$ to a constant, $|\phi|$ is constant $\mu$-a.e. Next, we rewrite $\Lambda\left(e^{i t h} \phi\right)=\lambda \phi$ as

$$
\frac{1}{d} \sum_{w \in f^{-1}(z)}\left(e^{i t h} \phi\right)(w)=\lambda \phi(z), \quad z \in \mathbb{P}^{1}
$$


Since $|\phi|$ is constant, this is only possible if

$$
e^{i t h(z)} \phi(z)=\lambda \phi(f(z)), \quad \mu \text {-a.e. } z \in \mathbb{P}^{1} .
$$

Since $h$ is not a cocycle, this implies that $\phi=0$ for $\mu$-a.e. $z \in \mathbb{P}^{1}$. Next, consider the function $\Lambda^{n}(|\phi|), n \in \mathbb{N}$. Since one has just shown that $\mu(|\phi|)=0$, it follows from (2.6) and the identity $\Lambda_{h}(i t)(\phi)=\lambda \phi$ that

$$
\|\phi\|_{L^{1}}=\left\|\Lambda_{h}^{n}(i t)(\phi)\right\|_{L^{1}}=\left\|\Lambda^{n}\left(e^{i t S_{n} h} \phi\right)\right\|_{L^{1}} \leq\left\|\Lambda^{n}(|\phi|)\right\|_{L^{1}} \leq \frac{C}{d^{n / 2}}\|\phi\|_{W^{1,2}} .
$$

Letting $n$ tend to $\infty$, we obtain $\phi=0$, which is a contradiction.

Now, assume that $\rho\left(\left.\Lambda_{h}(i t)\right|_{W^{1,2}}\right)<1$. Then for $\phi \in W^{1,2}$ we have $\Lambda_{h}^{n}(i t)(\phi) \rightarrow 0$ in $W^{1,2}$. It follows that $\Lambda_{h}^{n}(i t)(\phi) \rightarrow 0 \mu$-a.e. If $e^{i t h(z)} \phi(z)=e^{i s} \phi(f(z)) \mu$-a.e. then $\Lambda_{h}^{n}(i t)(\phi)=e^{i n s} \phi \mu$-a.e. Hence $\phi=0 \mu$-a.e. This implies that $h$ is not a multiplicative $W^{1,2}$-cocycle.

Now we are able to state the last main result.

Theorem C. We keep the hypothesis and the notation of Theorem A. Assume that $h$ is not a multiplicative $W^{1,2}$-cocycle. Then for every bounded interval $\Delta \subset \mathbb{R}$, the following convergence holds uniformly in $x \in \mathbb{R}$

$$
\lim _{n \rightarrow \infty}\left|\sigma \sqrt{n} \nu\left\{x+S_{n} h \in \Delta\right\}-\frac{1}{\sqrt{2 \pi}} e^{-\frac{x^{2}}{2 \sigma^{2} n}} m(\Delta)\right|=0
$$

where $m(\Delta)$ denotes the length of $\Delta$.

Proof. We follow the proof given by Breiman (see [2, pp. 224-227]) in the context of independant random variables. The proof of the above equality is reduced to showing that

$$
\lim _{n \rightarrow \infty}\left|\sigma \sqrt{n} \int_{\mathbb{P}^{1}} \phi\left(x+S_{n} h\right) \mathrm{d} \nu-\frac{1}{\sqrt{2 \pi}} \int_{-\infty}^{\infty} \phi(t) e^{-\frac{x^{2}}{2 \sigma^{2} n}} \mathrm{~d} t\right| \equiv \lim _{n \rightarrow \infty}\left|\frac{A_{n}(x)}{\sqrt{2 \pi}}\right|=0
$$

for every real-valued function $\phi \in L^{1}(\mathbb{R})$ whose Fourier transform $\widehat{\phi}(x):=$ $\frac{1}{\sqrt{2 \pi}} \int_{\mathbb{R}} \phi(t) e^{-i t x} \mathrm{~d} t$ is a continuous function supported in an interval $[-\delta, \delta], \delta>0$. 
Observe that

$$
\begin{aligned}
\sigma \sqrt{n} \int_{\mathbb{P}^{1}} \phi\left(x+S_{n} h\right) \mathrm{d} \nu & =\frac{\sigma \sqrt{n}}{\sqrt{2 \pi}} \int_{\mathbb{P}^{1}}\left(\int_{-\delta}^{\delta} \widehat{\phi}(t) e^{i t\left(x+S_{n} h\right)} \mathrm{d} t\right) g \mathrm{~d} \mu \\
& =\frac{\sigma \sqrt{n}}{\sqrt{2 \pi}} \int_{-\delta}^{\delta} \widehat{\phi}(t) e^{i t x}\left(\int_{\mathbb{P}^{1}} e^{i t S_{n} h} g \mathrm{~d} \mu\right) \mathrm{d} t \\
& =\frac{1}{\sqrt{2 \pi}} \int_{-\delta \sigma \sqrt{n}}^{\delta \sigma \sqrt{n}} \widehat{\phi}\left(\frac{t}{\sigma \sqrt{n}}\right) e^{\frac{i t x}{\sigma \sqrt{n}}}\left(\int_{\mathbb{P}^{1}} e^{i t \frac{S_{n} h}{\sigma \sqrt{n}}} g \mathrm{~d} \mu\right) \mathrm{d} t \\
& \left.=\frac{1}{\sqrt{2 \pi}} \int_{-\delta \sigma \sqrt{n}}^{\delta \sigma \sqrt{n}} \widehat{t} \frac{t}{\sigma \sqrt{n}}\right) e^{\frac{i t x}{\sigma \sqrt{n}}}\left(\int_{\mathbb{P}^{1}} \Lambda_{h}^{n}\left(\frac{i t}{\sigma \sqrt{n}}\right)(g) \mathrm{d} \mu\right) \mathrm{d} t
\end{aligned}
$$

We also need the following identities,

$$
\widehat{\phi}(0)=\frac{1}{\sqrt{2 \pi}} \int_{-\infty}^{\infty} \phi(t) \mathrm{d} t \quad \text { and } \quad e^{-\frac{x^{2}}{2 \sigma^{2} n}}=\frac{1}{\sqrt{2 \pi}} \int_{-\infty}^{\infty} e^{i t \frac{x}{\sigma \sqrt{n}}} e^{-\frac{t^{2}}{2}} \mathrm{~d} t .
$$

If we replace in (5.1) we obtain

$$
A_{n}(x)=\int_{-\delta \sigma \sqrt{n}}^{\delta \sigma \sqrt{n}} \widehat{\phi}\left(\frac{t}{\sigma \sqrt{n}}\right) e^{\frac{i t x}{\sigma \sqrt{n}}}\left(\int_{\mathbb{P}^{1}} \Lambda_{h}^{n}\left(\frac{i t}{\sigma \sqrt{n}}\right)(g) \mathrm{d} \mu\right) \mathrm{d} t-\widehat{\phi}(0) \int_{-\infty}^{\infty} e^{i t \frac{x}{\sigma \sqrt{n}}} e^{-\frac{t^{2}}{2}} \mathrm{~d} t .
$$

In order to estimate $A_{n}(x)$, we divide it into three pieces

$$
A_{n}(x) \equiv A_{n}^{1}(x)+A_{n}^{2}(x)+A_{n}^{3}(x) .
$$

They are given by the following formulas where the constant $\alpha \in(0, \delta)$ will be determined later on :

$$
\begin{aligned}
A_{n}^{1}(x) & :=\int_{-\alpha \sigma \sqrt{n}}^{\alpha \sigma \sqrt{n}} \widehat{\phi}\left(\frac{t}{\sigma \sqrt{n}}\right) e^{\frac{i t x}{\sigma \sqrt{n}}}\left(\int_{\mathbb{P}^{1}} \Lambda_{h}^{n}\left(\frac{i t}{\sigma \sqrt{n}}\right)(g) \mathrm{d} \mu\right) \mathrm{d} t-\widehat{\phi}(0) \int_{-\alpha \sigma \sqrt{n}}^{\alpha \sigma \sqrt{n}} e^{i t \frac{x}{\sigma \sqrt{n}}} e^{-\frac{t^{2}}{2}} \mathrm{~d} t, \\
A_{n}^{2}(x) & :=\int_{\alpha<\left|\frac{t}{\sigma \sqrt{n}}\right|<\delta} \widehat{\phi}\left(\frac{t}{\sigma \sqrt{n}}\right) e^{\frac{i t x}{\sigma \sqrt{n}}}\left(\int_{\mathbb{P}^{1}} \Lambda_{h}^{n}\left(\frac{i t}{\sigma \sqrt{n}}\right)(g) \mathrm{d} \mu\right) \mathrm{d} t, \\
A_{n}^{3}(x) & :=\widehat{\phi}(0) \int_{\alpha<\left|\frac{t}{\sigma \sqrt{n}}\right|} e^{i t \frac{x}{\sigma \sqrt{n}}} e^{-\frac{t^{2}}{2}} \mathrm{~d} t .
\end{aligned}
$$


To estimate $A_{n}^{1}(x)$ we recall from Theorem 3.2 that for $\left|\frac{t}{\sigma \sqrt{n}}\right|<a$

$$
\int_{\mathbb{P}^{1}} \Lambda_{h}^{n}\left(\frac{t}{\sigma \sqrt{n}}\right)(g) \mathrm{d} \mu=\lambda^{n}\left(\frac{t}{\sigma \sqrt{n}}\right) \int_{\mathbb{P}^{1}} \Phi\left(\frac{t}{\sigma \sqrt{n}}\right)(g) \mathrm{d} \mu+\int_{\mathbb{P}^{1}} \Psi^{n}\left(\frac{t}{\sigma \sqrt{n}}\right)(g) \mathrm{d} \mu .
$$

On the other hand, we know from the proof of Theorem A that

$$
\lim _{n \rightarrow \infty} \lambda^{n}\left(\frac{t}{\sigma \sqrt{n}}\right)=e^{-\frac{t^{2}}{2}}, \quad \lim _{n \rightarrow \infty} \int_{\mathbb{P}^{1}} \Phi\left(\frac{t}{\sigma \sqrt{n}}\right)(g) \mathrm{d} \mu=1, \quad \lim _{n \rightarrow \infty} \int_{\mathbb{P}^{1}} \Psi^{n}\left(\frac{t}{\sigma \sqrt{n}}\right)(g) \mathrm{d} \mu=0
$$

and there is a constant $\alpha>0$ (we can choose $\alpha<\delta$ ) such that for $\left|\frac{t}{\sigma \sqrt{n}}\right|<\alpha$,

$$
\begin{aligned}
\left|\lambda^{n}\left(\frac{t}{\sigma \sqrt{n}}\right) \int_{\mathbb{P}^{1}} \Phi\left(\frac{t}{\sigma \sqrt{n}}\right)(g) \mathrm{d} \mu\right|<C e^{-\frac{t^{2}}{4}}, \\
\left|\int_{\mathbb{P}^{1}} \Psi^{n}\left(\frac{t}{\sigma \sqrt{n}}\right)(g) \mathrm{d} \mu\right|<C \rho_{1}^{n}<C e^{-\frac{t^{2}}{4}} .
\end{aligned}
$$

Then,

$$
\left|\widehat{\phi}\left(\frac{t}{\sigma \sqrt{n}}\right) \int_{\mathbb{P}^{1}} \Lambda_{h}^{n}\left(\frac{i t}{\sigma \sqrt{n}}\right)(g) \mathrm{d} \mu-\widehat{\phi}(0) e^{-\frac{t^{2}}{2}}\right| \leq C e^{-\frac{t^{2}}{4}}\|\widehat{\phi}\|_{L^{\infty}(\mathbb{R})}, \quad \frac{t}{\sigma \sqrt{n}} \in[-\alpha, \alpha] .
$$

Observe that the right hand side of the latter estimate is an integrable function. On the other hand, since $\widehat{\phi}$ is continuous, we deduce from the previous identities that

$$
\lim _{n \rightarrow \infty} \widehat{\phi}\left(\frac{t}{\sigma \sqrt{n}}\right) \int_{\mathbb{P}^{1}} \Lambda_{h}^{n}\left(\frac{i t}{\sigma \sqrt{n}}\right)(g) \mathrm{d} \mu-\widehat{\phi}(0) e^{-\frac{t^{2}}{2}}=0, \quad t \in \mathbb{R} .
$$

Then the Lebesgue's dominated convergence theorem implies

$$
\lim _{n \rightarrow \infty}\left|A_{n}^{1}(x)\right|=0
$$

uniformly in $x \in \mathbb{R}$.

Now we estimate $A_{n}^{2}(x)$. For every $\tau \in[\alpha, \delta]$, consider the operator $T:=\Lambda_{h}(i \tau)$ : $W^{1,2} \rightarrow W^{1,2}$. Recall from Proposition 5.2 that $\rho\left(\Lambda_{h}(i \tau)\right)<1$. Using Proposition 3.1 and a compactness argument we may find $C>0$ and $0<\rho<1$ such that $\left\|\Lambda_{h}^{n}(i \tau)\right\| \leq C \rho^{n}, \tau \in[\alpha, \delta]$. Hence

$$
\left|\int_{\mathbb{P}^{1}} \Lambda_{h}^{n}\left(\frac{i t}{\sigma \sqrt{n}}\right)(g) \mathrm{d} \mu\right| \leq C^{\prime} \rho^{n}
$$

for $\alpha \leq\left|\frac{t}{\sigma \sqrt{n}}\right| \leq \delta$. Consequently, we deduce from (5.2) that

$$
\lim _{n \rightarrow \infty}\left|A_{n}^{2}(x)\right| \leq \lim _{n \rightarrow \infty} C^{\prime \prime} \sqrt{n} \rho^{n}=0
$$

uniformly in $x \in \mathbb{R}$. 
To estimate $A_{n}^{3}(x)$ it suffices to see that

$$
\lim _{n \rightarrow \infty}\left|A_{n}^{3}(x)\right| \leq \lim _{n \rightarrow \infty} \int_{\sqrt{n} \sigma \alpha<|t|} e^{-\frac{t^{2}}{2}} \mathrm{~d} t=0 .
$$

The estimates for $A_{n}^{1}(x), A_{n}^{2}(x)$ and $A_{n}^{3}(x)$ imply (5.1).

Acknowledgment. The second named author wishes to express his gratitude to the Max-Planck Institut für Mathematik in Bonn for its hospitality and support.

\section{REFERENCES}

[1] R. A. Adams and J. J. F. Fournier, Sobolev spaces, Second edition, Pure and Applied Mathematics, 140, Elsevier Science, 2003, vii+305 pp.

[2] L. Breiman, Probability, Addison-Wesley Publishing Company, Reading, Mass.-London-Don Mills, Ont. 1968 ix+421 pp.

[3] A. Broise, Transformations dilatantes de l'intervalle et théorèmes limites, in Études spectrales d'opérateurs de transfert et applications, Astérisque 1996, no. 238, 1-109.

[4] M. Denker M., F. Przytycki and M. Urbański, On the transfer operator for rational functions on the Riemann sphere, Ergodic Theory Dynam. Systems, 16 (1996), no. 2, 255-266.

[5] T.-C. Dinh and N. Sibony, Dynamique des applications d'allure polynomiale, J. Math. Pures et Appl., 82 (2003), 367-423.

[6] T.-C. Dinh and N. Sibony, Distribution des valeurs d'une suite de transformations méromorphes et applications, Comment. Math. Helv., 81 (2006), 221-258.

[7] T.-C. Dinh and N. Sibony, Decay of correlations and central limit theorem for meromorphic maps, Comm. Pure Appl. Math., to appear.

[8] D. Dolgopyat, On decay of correlations in Anosov flows, Ann. of Math. (2), 147 (1998), no. $2,357-390$.

[9] R. M. Dudley, Real analysis and probability, The Wadsworth \& Brooks/Cole Mathematics Series. Wadsworth \& Brooks/Cole Advanced Books \& Software, Pacific Grove, CA, 1989. xii+436 pp.

[10] N. Dunford and J. T. Schwartz, Linear operators. Part I. General theory, With the assistance of William G. Bade and Robert G. Bartle, Reprint of the 1958 original. Wiley Classics Library. A Wiley-Interscience Publication. John Wiley \& Sons, Inc., New York, 1988. xiv+858 pp.

[11] W. Feller, An introduction to probability theory and its applications. Vol. II., John Wiley \& Sons, Inc., New York-London-Sydney 1966 xviii+636 pp.

[12] J. E. Fornæss and N. Sibony, Complex dynamics in higher dimensions, Notes partially written by Estela A. Gavosto. NATO, Adv. Sci. Inst. Ser. C Math. Phys. Sci., 439, Complex potential theory (Montreal, PQ, 1993), 131-186, Kluwer Acad. Publ., Dordrecht, 1994.

[13] M. I. Gordin, The Central Limit Theorem for stationary processes, Soviet. Math. Dokl., 10 n.5 (1969), 1174-1176.

[14] N. Haydn, Convergence of the transfer operator for rational maps, Ergodic Theory Dynam. Systems, 19 (1999), no. 3, 657-669.

[15] E. Hebey, Nonlinear analysis on manifolds: Sobolev spaces and inequalities, Courant Lecture Notes in Mathematics, 5, New York University, Courant Institute of Mathematical Sciences, New York; American Mathematical Society, Providence, RI, 1999. x+309 pp.

[16] C. T. Ionescu Tulcea and G. Marinescu, Théorie ergodique pour des classes d'opérations non complètement continues, Ann. of Math., 52(2), (1950), 140-147.

[17] E. Le Page, Théorèmes limites pour les produits de matrices aléatoires, Probability measures on groups (Oberwolfach, 1981), pp. 258-303, Lecture Notes in Math., 928, Springer, BerlinNew York, 1982. 
[18] C. Liverani, Central limit theorem for deterministic systems, International Conference on Dynamical Systems (Montevideo, 1995), 56-75, Pitman Res. Notes Math. Ser., 362, Longman, Harlow, 1996.

[19] M. Ju. Lyubich, Entropy properties of rational endomorphisms of the Riemann sphere, Ergodic Theory Dynam. Systems, 3, (1983), no. 3, 351-385.

[20] S. V. Nagaev, Some limit theorems for Markov chains, Theor. Prob. Appl., 2, (1957), 378-416.

[21] M. F. Norman, Markov processes and learning models, Mathematics in Science and Engineering, vol. 84, Academic Press, New York-London, 1972. xiii+274 pp.

[22] F. Przytycki, On the Perron-Frobenius-Ruelle operator for rational maps on the Riemann sphere and for Hölder continuous functions, Bol. Soc. Brasil. Mat. (N.S.), 20, (1990), no. 2, 95-125.

[23] J. Rousseau-Egele, Un théorème de la limite locale pour une classe de transformations dilatantes et monotones par morceaux, Ann. Probab., 11(3), (1983), 772-788.

[24] N. Sibony, Dynamique des applications rationnelles de $\mathbb{P}^{k}$, Panoramas et Synthèses, 8 (1999), 97-185.

Tien-Cuong Dinh, Analyse Complexe, Plateau 7D, Institut de Mathématique de Jussieu, 175 Rue du Chevaleret, 75013 Paris, France

E-mail address: dinh@math.jussieu.fr

Viêt-Anh Nguyên, Max-Planck Institut für Mathematik, Vivatsgasse 7, D-53111, BOnN, GERMANY

E-mail address: vietanh@mpim-bonn.mpg.de

Nessim Sibony, Mathématique-BÂt 425, UMR 8628, Université Paris-Sud F-91405 ORSAY, FRANCE

E-mail address: nessim.sibony@math.u-psud.fr 